\title{
Resolving Bridging References in Unrestricted Text
}

\author{
Massimo Poesio, Renata Vieira and Simone Teufel \\ Centre for Cognitive Science - University of Edinburgh \\ 2, Buccleuch Place EH8 9LW Edinburgh UK \\ \{poesio, renata,simone\} @ogsci.ed.ac.uk
}

\begin{abstract}
Our goal is to develop a system capable of treating the largest possible subset of definite descriptions in unrestricted written texts. A previous prototype resolved anaphoric uses of definite descriptions and identified some types of first-mention uses, achieving a recall of $56 \%$. In this paper we present the latest version of our system, which handles some types of bridging references, uses WordNet as a source of lexical knowledge, and achieves a recall of $65 \%$.
\end{abstract}

\section{Previous Work}

We are in the process of developing a system for interpreting definite descriptions (DDs) in written text without restrictions of domain. The implementation work has been supported by an analysis of definite description use in corpora of written language (Poesio and Vieira, 1997). In one of our experiments, we asked 2 subjects to classify the uses of definite descriptions in a corpus of English texts ${ }^{1}$ using a taxonomy derived from the proposals of (Clark, 1977; Hawkins, 1978; Prince, 1981; Fraurud, 1990; Prince, 1992). In the taxonomy used in that study, we defined bridging references as those uses of definite descriptions based on previous discourse which require some reasoning in the identification of their textual antecedent (rather than just matching identical nouns). These definite descriptions may be coreferential with an entity already introduced in the discourse, but be characterized by a different head noun (as in a car ... the vehicle); or may be simply semantically related to it (in the sense that the door is related to house). Of the $1040 \mathrm{DDs}$ in that corpus, $204(20 \%)$ were identified as bridging descriptions, $312(30 \%)$ as anaphoric (DDs and antecedents

\footnotetext{
${ }^{1}$ A set of randomly selected parsed articles from the Wall Street Journal contained in the ACL/DCI CD-ROM.
}

which co-refer and have the same head noun), and $492(47 \%)$ as larger situation/unfamiliar (Hawkins, 1978) (Prince's discourse new (Prince, 1992)); the remaining definite descriptions were classified as idiomatic or doubtful cases.

These results led us to concentrate initially on resolving same-head anaphoric DDs and on recognising larger situation/unfamiliar uses. Our analysis of the corpus suggested that many of the latter could be recognised using syntactic heuristics: e.g., on the basis of the presence of restrictive pre- and postmodification, of the presence of special predicates (such as the superlatives first, best), or because the DD occurred in a copula or appositive construction.

A first prototype with these capabilities (Vieira and Poesio, 1997) achieved an overall recall of $56 \%$ and precision of $84 \%$ when tested on our corpus. Of all anaphoric DDs $72 \%$ were resolved, and $74 \%$ of all larger situation and unfamiliar uses were identified. The definite descriptions not handled by this first prototype were typically larger situation uses based on common knowledge (such as the government) and bridging descriptions. In this paper we present our subsequent work devoted to handling some of these remaining cases.

\section{Bridging Descriptions}

Linguistic and computational theories of bridging descriptions identify two main subtasks involved in their resolution: first, finding the element in the text to which the bridging description is related (ANCHOR) and second, finding the relation (LINK) holding between the bridging description and its anchor (Clark, 1977; Sidner, 1979; Heim, 1982; Carter, 1987; Fraurud, 1990; Strand, 1997).

A speaker is licensed to use a bridging DD when he/she can assume that the common-sense knowledge required to identify the relation is shared by the listener (Hawkins, 1978; Clark and Marshall, 1981; Prince, 1981). This reliance on commonsense 
knowledge means that, in general, a system could only resolve bridging references when supplied with an adequate knowledge base; for this reason, the typical way of implementing a system for resolving bridging references has been to restrict the domain and feeding the system with hand-tailored world knowledge. (This approach is discussed in detail in (Carter, 1987)). In order to get a system capable of performing on unrestricted text, we decided to use WordNet (WN) (Miller, 1993) as an approximation of a knowledge base containing generic information, and to supplement it with heuristics to handle those cases which WN couldn't handle.

Vieira and Teufel (1997) analyzed the corpus to identify the cases of bridging descriptions that could be resolved using WordNet, those for which we could use heuristics, and those that couldn't be interpreted at the moment. Six classes of bridging descriptions were identified. ${ }^{2}$

Synonymy/Hyponymy/Meronymy This class (henceforth, Syn/Hyp/Mer) includes those DDs which are in a synonymy/hyponymy/meronymy relation with their anchors, i.e., the kind of semantic relation that is currently encoded in WordNet. Examples ${ }^{3}$ are:

(1) a. Synonymy: new album - the record; three bills - the legislation.

b. Hypernymy-Hyponymy: rice - the plant; the daily television show - the program.

c. Meronymy (part-of relation): plants - the pollen; house - the chimney.

Names This class includes definite descriptions that refer back to proper names such as people's and company names, as in:

(2) Bach - the composer; Pinkerton's Inc - the company.

Compound Nouns This class includes bridging descriptions whose LINGUISTIC ANCHOR (i.e., the element in the text to which they are related) is a noun occurring as part of a compound noun other than the head. Examples include:

(3) stock market crash - the markets; discount packages - the discounts.

Events These are cases where the linguistic anchors of DDs are not NPs but VPs or sentences. Examples are:

\footnotetext{
${ }^{2}$ We should stress that this classification is primarily motivated by processing considerations.

${ }^{3}$ Note that the examples in (1a) and (1b) are classified as bridging even though the relation is of co-reference.
}

\begin{tabular}{|l|l|l|}
\hline Class & Total & $\%$ \\
\hline \hline Syn/Hyp/Mer & $12 / 14 / 12$ & $19 \%$ \\
\hline Names & 49 & $24 \%$ \\
\hline Compound Nouns & 25 & $12 \%$ \\
\hline Events & 40 & $20 \%$ \\
\hline Discourse Topic & 15 & $7 \%$ \\
\hline Inference & 37 & $18 \%$ \\
\hline Total & 204 & $100 \%$ \\
\hline
\end{tabular}

Table 1: Distribution of types of bridging DDs

(4) Individual investors and professional money managers contend. - They make the argument ...; Kadane Oil Co. is currently drilling two wells and putting money into three others. - The activity...

Discourse Topic There are some cases of DDs which are related to the (often implicit) discourse topic (in the sense of (Reinhart, 1981)) of a text, rather than to some specific NP or VP. For instance,

(5) the industry (in a text whose discourse topic is oil companies); the first half (in a text whose discourse topic is a concert).

Inference We collect in this class all the cases of bridging descriptions whose relation with their NP anchor was based on more complex inferential relations: for example, cases in which the relation between the anchor and the DD was of reason, cause, consequence, or set-membership:

(6) last week's earthquake - the suffering people are going through; Democratics/Republicans - the two sides.

The relative importance of these classes in our corpus is shown in Table 1. This classification is based on what we took to be the main linking relation for each of the 204 bridging DDs in the corpus ${ }^{4}$.

\section{Resolution of Bridging Descriptions}

We used Vieira and Teufel's analysis as the basis for the implementation of a second prototype. In this section we discuss how this prototype handles the different types of bridging descriptions.

\subsection{Bridging DDs and WordNet}

We implemented a WordNet interface that reports a possible semantic link between two nouns when one

\footnotetext{
${ }^{4}$ One problem with bridging references is that they are often related to more than one antecedent in the discourse (Poesio and Vieira, 1997).
} 
of the following is true:

- The nouns are in the same synset (= synonyms of each other), as in suit - lawsuit.

- The nouns are in direct hyponymy relation with each other, for instance, dollar - currency.

- There is a direct or indirect meronymy between them. Indirect meronymy holds when a concept inherits parts from its hypernyms, like car inherits the part wheel from its hypernym wheeled_vehicle.

- Due to WordNet's idiosyncratic encoding, it is often necessary to look for a semantic relation between coordinate sisters, i.e. hyponyms of the same hypernym, such as home - house which are hyponyms of housing, lodging.

Sometimes, if a relation between two head nouns is not encoded in WN directly, the semantic closeness might be found through the compound nouns made up of them. Thus, for a pair such as record, album we find synonymy between record_album and album.

We ran a test in which WordNet was used to identify the DD's anchors. For each of the 204 bridging DDs in our corpus, we considered the NPs in the previous five sentences as a potential anchor ${ }^{5}$, and queried WN with the DD and potential anchor pair.

WordNet reported a possible relation for 107 of the 204 DDs. Often, more than one link was found between a DD and a potential anchor, ${ }^{6}$ and sometimes links could be found with more than one potential anchor in the previous five sentences. Only in 34 of these 107 cases we found at least one appropriate anchor linking relation, and only in 21 cases we found only appropriate anchors (for 13 there was a mixture of suitable and unsuitable anchors).

Of these 34 DDs for which a correct anchor was found, only 18 were among those we had classified as Syn/Hyp/Mer. In 8 of these 18 cases, WordNet found a link with an anchor that was not the one we had identified manually, but which was still valid; for instance, we identified the link pound - the currency which was not found in WordNet, whereas our automatic search found sterling - the currency).

\footnotetext{
${ }^{5}$ Our system does not currently include a proper segmentation algorithm. Instead, we use a simple recency heuristic-we only consider the antecedents in the $n$ previous sentences, where $\boldsymbol{n}$ is a constant determined empirically. In our previous work (Vieira and Poesio, 1997). we observed that 5 was the value of $n$ which gave the best tradeoff between precision and recall.

${ }^{6}$ For instance, transaction and trade were reported to be in a hypernym relation and were also reported as coordinate sisters having as common hypernymy commerce.
}

The 16 remaining relations were found for DDs that we had not classified as Syn/Hyp/Mer: for instance, whereas we had classified the DD the population as belonging to the class of DDs based on names having as anchor Koreans, our implementation found a WordNet relation for the pair nation - the population; the system also found a few relations with proper names, such as Bach - the composer.

In the following tests we have considered only direct meronymy, as indirect meronymy presented extreme low recall and precision at a very expensive computational cost.

In order to reduce the number of false positives (86 out of 107$)^{\top}$, we tried using a stack-based approach towards finding potential anchors in the previous sentences, as suggested in (Sidner, 1979); i.e., the system would go back one sentence at a time, and stop as soon as a relation with a potential anchor was found, rather than trying to find all possible links. As a result, we found exactly one correct anchor for 30 DDs, slightly improving our results (76 false positives).

\begin{tabular}{|l|l|l|l|l|}
\hline Class & Total & Right & Wrong & P \\
\hline \hline Syn & 11 & 4 & 7 & $36 \%$ \\
\hline Hyp & 59 & 18 & 42 & $30 \%$ \\
\hline Mer & 6 & 2 & 4 & $33 \%$ \\
\hline Sister & 30 & 6 & 24 & $20 \%$ \\
\hline Total & 106 & 30 & 76 & $28 \%$ \\
\hline
\end{tabular}

Table 2: Analysis of the anchors found in WN

Table 2 shows the distribution of the different semantic relations between DDs and the anchors found by our stack-based search. It presents precision figures (P) related to each type of relation ${ }^{8}$. Sister relations are the least satisfactory ones.

We tested, in particular, whether WordNet encoded a semantic link between the 38 syn/hyp/mer relations in our corpus (just described) plus other 32 relations extracted from a second corpus study (Poesio and Vieira, 1997). The results for the 70 DDs are summarised in Table 3 . Overall recall (R) was $46 \%$. We could have expected $100 \%$ precision, since we had manually identified the anchors, but the precision figures $(\mathrm{P})$ report an error when a sister relation is found instead of the expected (syn/hyp/mer) re-

\footnotetext{
${ }^{7}$ The anchors found for 73 of the DDs were incorrect, for the remaining 13 DDs the resulting anchors were mixed (some right/some wrong).

${ }^{8}$ We cannot estimate recall since we do not have a precise number of syn/hyp/mer anchors that should be found (as different types of anchors may allow resolution of bridging descriptions)
} 
lation. The poorest recall was surprisingly obtained for synonymy relations, followed by meronymy relations, as expected, since these are only partially implemented in WordNet.

\begin{tabular}{|l|l|l|l|l|l|}
\hline Class & Total & In WN & Out & R & P \\
\hline \hline Syn & 20 & 7 & 13 & $35 \%$ & $71 \%$ \\
\hline Hyp & 32 & 18 & 14 & $56 \%$ & $94 \%$ \\
\hline Mer & 18 & 7 & 11 & $38 \%$ & $71 \%$ \\
\hline Total & 70 & 32 & 38 & $46 \%$ & $84 \%$ \\
\hline
\end{tabular}

Table 3: Search for semantic relations in WN

The low recall for synonymy relations may be due to the context dependent, specialized senses of sublanguage terminology (for instance, crash, bust and slump in Economics terminology). Some nouns were not even encoded in WN (such as newsweekly, spinoff). Other relations were missed due to the unexpected way in which knowledge is organised in WordNet. For example, no association was found between house and walls, because house is not encoded in WordNet as a hyponym of building but of housing, and housing does not have a meronymy link to wall whereas building does. Another example of counterintuitive position in the hierarchy is that of catastrophe, not listed as a hypernym of earthquake, but as its coordinate sister. This example demonstrates the problems that WordNet lexicographers faced when they had to coerce real-world concepts into a hierarchy with single, no-default inheritance: catastrophes clearly subclassify into earthquakes and floods, but minor earthquakes don't have to be catastrophic.

\subsection{Proper names}

Definite descriptions which refer back to proper names are very common in Wall Street Journal articles. Processing such DDs involves, first, determining an entity type for each name in the text, then searching for semantic relations. If we get the entity type person for the a name such as Mrs. Y.J. Park we could, ideally, resolve the subsequent DD the housewife using WordNet. A few names are available in WordNet: typically, famous people, countries, states, cities and languages. Other entity types can be identified using appositive constructions and abbreviations like $M r$., Co., Inc. etc. as cues.

The algorithm we developed, based on a mixture of access to WordNet and heuristics such as those we described, found the correct type for $66 \%$ of the names in our corpus (535/814). Including a backtracking mechanism which re-processes a text looking for missing name types (with this mechanism we identify the type for the name Morishita in a textual sequence like Morishita - Mr. Morishita) increases our recall to $69 \%(562 / 814)$. We then used WordNet to match the types found with previous references in the text. This resulted in the resolution of $53 \%$ $(26 / 49)$ of the cases based on names. We missed relations which are not found in WordNet (for instance, Mr. Morishita - the 57 year-old). But again we also found a large number of false positives.

\subsection{Compound Nouns}

Sometimes, a bridging description is linked to a nonhead noun in a compound noun:

stock market crash - the markets; rule changes - the rules; discount packages - the discounts.

One way of processing these definite descriptions would be to update the discourse model with discourse referents not only for the NP as a whole, but also for the embedded nouns: for example, after processing stock market crash, we could introduce a discourse referent for stock market, and another discourse referent for stock market crash. The DD the markets would be co-referring with the first of these referents (with identical head noun), and we could simply use our anaphora resolution algorithms. This solution, however, makes available discourse referents that are generally unaccessible for pronominal anaphora. We therefore followed a different route: our algorithm for identifying antecedents attempts to match not only heads with heads, but also the head of a DD with the pre-modifiers of a previous $\mathrm{NP}$, the pre-modifiers of a DD with the pre-modifiers of its antecedents, and the pre-modifiers of the DD with the head of a previous NP. With this, we obtained a total of 54 resolutions of which 36 were correct, although we did not always find a main linking relation licensing the use of a DD.

Examples of correct resolutions are:

(8) a. Head of DD with pre-modifier of antecedent: the stock market crash - the markets; rule changes - the rules;

b. Pre-modifiers of DD with pre-modifiers of antecedent: most oil companies - the oil fields; his art business - the art gallery;

c. Pre-modifiers of DD with head of antecedent: New York City - the city council district lines; a 15-acre plot and main home - the home site.

There are also cases in which the pre-modifiers plus the head noun of a DD may indicate a bridging reference: we may find an antecedent with the same head noun for a DD but referring to a different 
entity, this being signalled by the pre-modification. Some examples:

(9) the company's abrasive segment - the engineering materials segment; Italy's unemployment rate - the southern unemployment rate; Pinkerton - the new Pinkerton; increases of $3.9 \%$ - the actual wage increases may have been bigger.

Our previous heuristics for treatment of premodifiers in anaphoric resolution handled the first two examples correctly (Vieira and Poesio, 1997): as they present different pre-modifiers we did not treat them as anaphoric in the first version of our system. Such cases, as well as DDs modified by new and actual (last two examples), may now be treated as bridging references ${ }^{9}$.

\subsection{Events}

To process DDs based on events (situations or propositions), we are trying, as a first approach, to transform verbs into their nominalizations, and then looking for a relation in WordNet. Some nominalizations can be generated by general procedures or learned by means of a stochastic method: e.g., we could use WordNet's morphology component as a stemmer, and augment the verbal stems with the most common suffixes for nominalizations which could be kept in a list, like -ment, -ion. In our corpus, $17 \%(7 / 40)$ of the bridging references based on events are direct nominalizations of this type (for instance, changes were proposed - the proposals).

Another $17 \%$ are cases in which knowledge of the semantics of the verb is necessary (as in borrowed the loan). The remaining $66 \%$ (26 cases) of bridging DDs based on events require inference reasoning based on the compositional meaning of the phrases (as in It went looking for a partner - pitching the prospect); these cases are out of reach just now, as well as the cases listed under "discourse topic" and "inference".

\section{Restrictive Post-modification as Anchors}

Whereas the problem of finding the appropriate textual anchor for bridging descriptions requires knowledge inference and reasoning, DDs with restrictive post-modification give the reader both anchor and description in the same expression. As Hawkins points out (Hawkins, 1978):

... first mention uses of the with both referent establishing relative clauses and as-

\footnotetext{
${ }^{9}$ This idea is not implemented yet.
}

sociative clauses are not essentially different from the other uses mentioned in the last section (Hawkin's associative uses/our bridging uses). The only difference is that in the latter uses set identification and the locatability of the referent were possible on account of previous triggers, whereas it is now function of the modifier itself to provide the information which makes set identification and location possible(parentheses ours).

This could be seen as an advantage, as we directly find the anchors of these DDs. They are quite common uses of DDs, but not much attention has been devoted to them as a special case of anchor linking relations. The main problem for these cases is to find out their links, which is also a remaining problem for our proposals of anchor identification throughout the paper.

The head noun of a DD and its modifier may be related in different ways, as shown by the examples:

(10) the number of job seekers; the anthers of the plant; the ideal of a level playing field; the flip side of the Stoltzman personality.

In some cases, the relation between noun and complement seem to be looser than the relations for bridging descriptions. Sequences such as the laus of heredity; the cost of the plan are acceptable, whereas heredity - the laws; the plan - the cost are unlikely to occur. On the other hand, bridging such as the house - the kitchen; the firm - the owners are as acceptable as the kitchen of the house; the owners of the firm. Some proposals of a systematic treatment for the identification of anchor linking relations for bridging DDs are (Heim, 1982; Barker, 1991; Poesio, 1994; Strand, 1997).

\section{Discussion}

We presented our most recent results concerning the resolution of bridging descriptions. We identified different types of bridging descriptions, and we proposed a treatment for each of them separately.

We presented some preliminary experiments with WordNet. Our experience was mixed. WordNet was useful in determining the type of entity for some of the proper names in our corpus, typically cities, states and countries. On the other hand, Word. Yet proved to be unreliable for the automatic identification of possible link relations, as too many false positives were found. A 'blind' WordNet search for semantic relations is also very expensive computationally. A mechanism for focus tracking (Grosz and 
Sidner, 1986) or a clustering algorithm should be applied first in order to minimise the costs.

In order to have proper names available for resolution of future references, it is useful to create discourse referents for them which contain their entity types. Up to now we have identified an entity type for $69 \%$ of the names in our corpus, and we resolved $53 \%$ of the DDs referring back to proper names with the help of WordNet.

Pre-modifiers in compound nouns may license the use of definite descriptions. We have presented some preliminary tests that should be further developed.

Cases of bridging references based on events or propositions usually involve common-sense reasoning; some of them (in our corpus, $34 \%$ of all cases based on events) can however be solved by transforming verbs into their nominalizations and then searching for a semantic relation.

We also claimed that the same problem of determining a linking relation for bridging descriptions holds for first mention uses of DD based on restrictive post-modification.

As an estimate, we could say that about $60 \%$ of the cases in the bridging class could be treated by developing the ideas proposed here. We combined the proposed heuristics with the first version of our system - we applied the heuristics for proper names, compound nouns and WordNet consult (in this order) to the cases which the system did not handle before. The impact on the overall system performance was an increase in recall from $56 \%$ to $65 \%$ (note that the bridging class is a small class compared to the others) but precision decreased from $84 \%$ to $82 \%$. The heuristics should be further developed, and their integration into the system should be worked out in more detail-the heuristics could be implemented in parallel or through a decision tree.

Acknowledgements The authors would like to thank Kjetil Strand and the anonymous referees for their comments on earlier drafts of the paper. The authors are supported by an EPSRC Advanced Fellowship, a CNPq studentship and an EPSRC studentship, respectively.

\section{References}

Barker, C. 1991. Possessive Descriptions. Ph.D. thesis, University of California at Santa Cruz, Santa Cruz, CA.

Carter, D. M. 1987. Interpreting Anaphors in Natural Language Texts. Ellis Horwood.

Clark, H. H. 1977. Bridging. In P. N. JohnsonLaird and P.C. Wason, eds., Thinking: Readings in Cognitive Science. Cambridge University Press.
Clark, H. H. and C. R. Marshall. 1981. Definite reference and mutual knowledge. In Elements of Discourse Understanding. Cambridge University Press.

Fraurud, K. 1990. Definiteness and the processing of NPs in natural discourse. Journal of Semantics, $7: 395-433$.

Grosz, B. J. and C. L. Sidner. 1986. Attention, intention, and the structure of discourse. Computational Linguistics, 12(3):175-204.

Hawkins, J. A. 1978. Definiteness and Indefiniteness. Croom Helm.

Heim, I. 1982. The Semantics of Definite and Indefinite Noun Phrases. Ph.D. thesis, University of Massachusetts at Amherst.

Miller, G. et al. 1993. Five papers on WordNet. Technical Report CSL Report 43, Cognitive Science Laboratory, Princeton University.

Poesio, M. 1994. Weak definites. In Proceedings of the Fourth Conference on Semantics and Linguistic Theory, SALT-4. Cornell University Press.

Poesio, M. and Vieira, R. 1997. A Corpus based investigation of definite description use. Manuscript, Centre for Cognitive Science, University of Edinburgh.

Prince, E. F. 1981. Toward a taxonomy of givennew information. In P. Cole, ed., Radical Pragmatics. Academic Press, pages 223-256.

Prince, E. F. 1992. The ZPG letter: subjects, definiteness, and information status. In S. Thompson and W. Mann, eds., Discourse description: diverse analyses of a fund-raising text. John Benjamins, pages $295-325$.

Reinhart, T. 1981. Pragmatics and Linguistics: An Analysis of Sentence Topics Philosophica, 27(1).

Sidner, C. L. 1979. Towards a computational theory of definite anaphora comprehension in English discourse. Ph.D. thesis, MIT.

Strand, K. 1997. A Taxonomy of Linking Relations. Journal of Semantics, forthcoming.

Vieira, R. and M. Poesio. 1997. Processing definite descriptions in corpora. In S. Botley and M. McEnery, eds., Corpus-based and Computational Approaches to Discourse Anaphora. UCL Press.

Vieira, R. and S. Teufel. 1997. Towards Resolution of Bridging Descriptions. In Proceedings of the ACL Student Session. 